\title{
Learned value and predictiveness affect gaze but not figure assignment
}

\author{
Sandersan Onie ${ }^{1,2} \cdot$ Mary A. Peterson $^{3} \cdot$ Mike Le Pelley $^{1} \cdot$ Steven B. Most ${ }^{1}$
}

Published online: 3 November 2020

(C) The Psychonomic Society, Inc. 2020

\begin{abstract}
Many factors affect figure-ground segregation, but the contributions of attention and reward history to this process is uncertain. We conducted two experiments to investigate whether reward learning influences figure assignment and whether this relationship was mediated by attention. Participants learned to associate certain shapes with a reward contingency: During a learning phase, they chose between two shapes on each trial, with subsets of shapes associated with high-probability win, low-probability win, high-probability loss, and low-probability loss. In a test phase, participants were given a figure-ground task, in which they indicated which of two regions that shared a contour they perceived as the figure (high-probability win and low-probability win shapes were pitted against each other, as were high-probability loss and low-probability loss shapes). The results revealed that participants had learned the reward contingencies and that, following learning, attention was reliably drawn to the optimal stimulus. Despite this, neither reward history nor the resulting attentional allocation influenced figure-ground organization.
\end{abstract}

Keywords Learned value $\cdot$ Predictiveness $\cdot$ Visual perception $\cdot$ Figure-ground perception $\cdot$ Eye gaze $\cdot$ Attention

The world is filled with ambiguity. Consider visual input, which typically consists of many regions abutting each other like a mosaic. Perceptual organization processes must determine which parts of the input constitute objects and which are their backgrounds. Until these roles are assigned, there is ambiguity at every border between two regions. One common outcome of perceptual organization is that the shared border is seen as belonging to one region: This region is perceived as a shaped figure or object, bounded by the border, whereas the other region is perceived as a shapeless background that seems to continue behind the figure/object. One famous example of figureground perception is depicted in Fig. 1, also known as the Rubin Vase/Faces stimulus (Rubin, 1915). When the central white region is perceived as the figure/object, it appears to have a definite shape - that of a vase; the black regions appear shapeless near the border of the vase and seem to simply continue behind it. In contrast, when the black regions appear to be figures, they seem to have a definite shape - that of two profiles facing

Sandersan Onie

sandy.onie@gmail.com

UNSW Sydney, Sydney, Australia

Black Dog Institute, Sydney, Australia

3 University of Arizona, Tucson, AZ, USA toward the center; when the face profiles are perceived, the central white region appears shapeless near the contours of the face profiles and seems to continue behind them.

Myriad priors (formerly called "cues") affect figure assignment, including symmetry, closure, small area, and convexity (Bahnsen, 1928; Goldreich \& Peterson, 2012; Hoffman \& Singh, 1997; Kanizsa \& Gerbino, 1976; Peterson \& Salvagio, 2008; Rubin, 1915). The region on the side of the border with these properties is more likely to be perceived as the figure/object than the other side, which may lack these properties. In addition, past experience (e.g., the suggestion of portions of familiar objects on one side of a border shared by two regions) can guide which region is perceived as the bounded object (e.g., Peterson, 1994; Peterson \& Gibson, 1994a; Peterson, Harvey, \& Weidenbacher, 1991; Navon, 2010; Vecera \& Farah, 1997). Evidence that experience contributes to the perception of objecthood (i.e., is an object prior) is important because it shows that learning affects figure assignment. Initially, the other aforementioned priors were hypothesized to be innate (e.g., Koffka, 1935; Wertheimer, 1923/1938), although we note that they too may arise from learning (e.g., objects in the world tend to be symmetric, have convex parts; Jacobs, 1996; Livio, 2005; Pizlo, 2010).

Evidence has been mixed about the role of another potential learned prior on figure/ground assignment: the learned value of figures/objects. For instance, Schafer and Murphy (1943) 


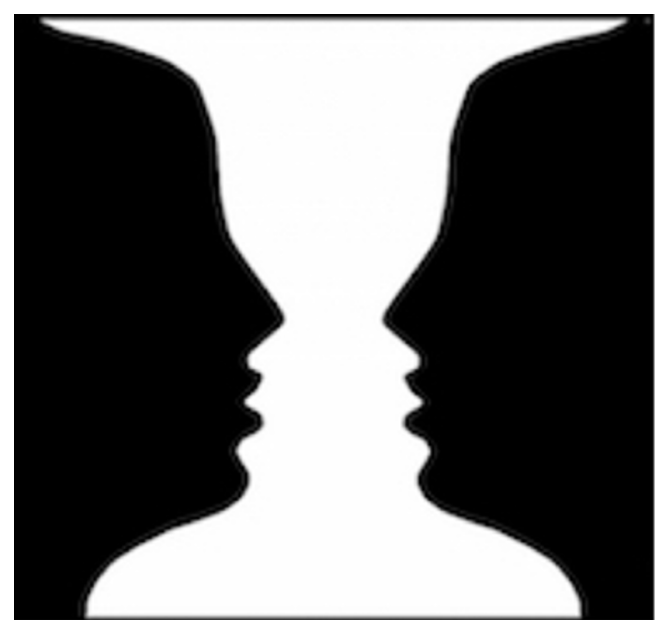

Fig. 1 The Rubin Vase. A classic example of figure-ground segregation in which either a vase or two faces are consciously perceived, depending on whether the white or black region (respectively) is perceived as the shaped figure

rewarded participants with small amounts of money when they viewed two stylized outline drawings of face profiles ( +2 cents or +4 cents), and punished them by taking away money when they viewed two other face profiles ( -2 cents or -4 cents). Participants $(N=5)$ later viewed composite stimuli in which a rewarded face profile shared a border with a punished face profile and were asked to report the side on which they perceived the figure. Participants reported perceiving the figure on the side of the border containing the rewarded face on $68 \%$ of trials, which was significantly above chance (50\%). Accordingly, Schafer and Murphy concluded that learned value affects figure assignment. However, using the same stimuli and a very similar procedure, Rock and Fleck (1950) failed to replicate Schafer and Murphy's results: their participants $(N=6)$ reported perceiving rewarded face profiles as the figures on only $42 \%$ of trials. To increase the motivational salience of differences in relative value, Smith and Hochberg (1954) eschewed small monetary rewards and punishments, and instead paired two of the face profiles with electric shock; the other two were not paired with shock. In the composite stimuli used at test, a face profile that was paired with shock (punished) during learning shared a border with one that was not paired with shock. Like the previous investigators, Smith and Hochberg balanced which specific face profiles were punished between small groups of participants. At test, one group $(N=5)$ perceived the figure on the unshocked side of the border on $73 \%$ of trials, whereas the other group $(N=$ 5 ) perceived the figure on the unshocked side of the border on only $47 \%$ of trials. Therefore, there is mixed evidence for the effect of reward on figure-ground perception. Note, however, that the previous experiments may not have been sensitive tests of the question: The numbers of participants were small, and there were no independent assays of how well participants had learned the reward-punishment associations. Moreover, Smith and Hochberg determined that the exposure duration used at test
( $\sim 333 \mathrm{~ms}$ ) was long enough for participants to perceive both face profiles on nearly half of trials. Hence, the possibility remains that when effects of value on figure assignment were present, participants may not have reported their initial percept.

In contrast to the mixed results about whether learned value affects figure assignment, reliable effects have been reported on attentional selection (e.g., Chelazzi, Peralto, Santandrea, \& Libera, 2013; Lee \& Shomstein, 2014; Pessoa, 2015). A recent systematic review (Le Pelley, Mitchell, Beesley, George, \& Wills, 2016) highlighted a body of literature demonstrating that stimuli that have - in the past - been experienced as being more predictive of a valenced outcome (whether positive or negative) reliably attract greater attention than stimuli experienced as nonpredictive. That is, attention is influenced by learning about predictiveness - the extent to which stimuli provide information about upcoming events. Predictiveness has been shown to attract attention both in spatial paradigms (e.g., Le Pelley, Beesley, \& Griffiths, 2009; Le Pelley, Vadillo, \& Luque, 2013; Rehder \& Hoffman, 2005) and nonspatial attention paradigms (e.g., Livesey, Harris, \& Harris, 2009; Luque, Morís, Rushby, \& Le Pelley, 2015), and to influence the rate of future learning (e.g., Beesley \& Le Pelley, 2010; Bonardi, Graham, Hall, \& Mitchell, 2005; Griffiths \& Le Pelley, 2009; Le Pelley \& McLaren, 2003). Furthermore, stimuli that have more value are also more likely to attract attention than less valuable stimuli. This holds true for intrinsically valuable stimuli (e.g., food in obese individuals; Hendrikse et al., 2015) and also for neutral stimuli paired with a valuable outcome during an experiment (e.g., learning that a red circle signals monetary reward increases the likelihood that red circles will attract attention in future; for examples, see Anderson, Laurent, \& Yantis, 2011; Kahnt, Park, Haynes, \& Tobler, 2014; Le Pelley, Pearson, Griffiths, \& Beesley, 2015; Le Pelley, Seabrooke, Kennedy, Pearson, \& Most, 2017; O'Brien \& Raymond, 2009). Many of these studies used eyetracking to measure attention: Gaze provides an excellent online index of attention because an eye movement to a given location is always preceded by a shift of attention to that location (Deubel \& Schneider, 1996; Hoffman \& Subramaniam, 1995; Kowler Anderson, Dosher, \& Blaser, 1995). For this reason, eye movements are often referred to as shifts of overt attention.

The attention effects reviewed above suggest a potential mechanism whereby learning about value and/or predictiveness might influence figure assignment. This is because research suggests that figures are more likely to be perceived on the side of a border where attention is allocated. These effects have been observed when attention is allocated both endogenously (Baylis \& Driver, 1995; Driver \& Baylis, 1996) and exogenously (Vecera, Flevaris, \& Filapek, 2004). Peterson and Gibson (1994b) found similar effects for gaze fixation (which, as noted above, indexes attention).

Overall, then, we have evidence that (1) learning about value affects attentional selection and (2) that attended regions are likely to be seen as figures. Hence, the idea that learned value 
might influence figure assignment has surface plausibility and is worthy of further and more rigorous investigation beyond the earlier studies reported in the object perception literature (Rock \& Fleck, 1950; Schafer \& Murphy, 1943; Smith \& Hochberg, 1954). This was the rationale for the current study, in which we investigated whether effects of learned value and/or predictiveness (independent of value) on figure assignment would be observed in experiments (a) with a larger number of participants than were tested in the 1940s and 1950s when this question was previously investigated, and (b) in which we used a learning criterion to ensure that participants had indeed learned from their experiences before effects of value on figure assignment were assessed.

Our study had two distinct phases. In the first phase, we paired shapes with rewards, and in the second phase, we investigated whether effects of reward-learning from the first phase influenced figure-ground segregation.

In the first phase of our task, we adapted methods used by others to associate stimuli with rewards and losses (O'Brien \& Raymond, 2012; Raymond \& O'Brien, 2009). On each trial, participants selected a shape from a shape pair with the goal of earning points (which translated to monetary rewards; Fig. 2 shows example stimuli). There were two trial types: "win" trials and "loss" trials. On win trials, selection of one stimulus was associated with a $90 \%$ chance of winning points, whereas the other was associated with a $10 \%$ chance of winning points (and a $90 \%$ chance of winning nothing). On loss trials, selecting one stimulus was associated with a $90 \%$ chance of losing points, whereas the other was associated with a $10 \%$ chance of losing points. In total, there were eight shapes, formed into four pairs, with two "win" and two "loss" pairs. Shape pairings and associated reward contingencies were held constant throughout the study, with reward contingencies counterbalanced across participants. This procedure allowed us to contrast value and predictiveness. "Value" in this task relates to the optimal choice in each trial: This is determined by what choice will—on average - result in the most points (on win trials, the optimal choice is the shape giving a $90 \%$ chance of reward; on loss trials, the optimal choice is the shape giving a $10 \%$ chance of loss). "Predictiveness" relates to the probability that choosing a particular shape produces an outcome (win or loss of points) versus no outcome (no win or loss): high-probability stimuli are those paired with a $90 \%$ chance of winning points (on win trials) or losing points (on loss trials), and low-predictive stimuli are those paired with a $10 \%$ chance of winning or losing points. Therefore, stimuli that were high in value were not necessarily high in probability, and vice versa. We recorded eye-tracking data throughout this first phase, which allowed us to examine the effect of participants' learning about the different shapes on their overt attention to those shapes. While we would expect participants to come to pay more attention to optimal stimuli than suboptimal stimuli in both win and loss trials, we hypothesized that the particular pattern and dynamics of this attentional bias toward optimal stimuli might differ in win trials (in which the optimal choice was the high-probability stimulus) versus loss trials (in which the optimal choice was the low-probability stimulus). Any such difference would indicate that the learning task induced changes in attention as a function of both value and predictiveness.

In the second phase, we assessed whether prior learning about value or predictiveness affected figure assignment. For this purpose, we used two composite "win" stimuli (in which a portion of a high-probability win stimulus shared a border with a portion of a low-probability win stimulus) and two composite "loss" stimuli (in which a portion of a high-probability loss stimulus shared a border with a portion of a low-probability loss stimulus). This method of creating the figure-ground test stimuli preserved the borders of the stimuli learned during the first phase, where participants associated stimuli with reward/loss, to the second phase. If value and/or predictiveness affect figure assignment, then on "win" composite test stimuli, participants should report perceiving the figure on the side of the border where a portion of the high-probability win stimulus is sketched (since this stimulus was high in both probability and value). Behavior on "loss" composite test stimuli would in principle allow us to disentangle whether learning about value or predictiveness was the driving force behind any observed effects (here, we follow a rationale used in prior research; see O'Brien \& Raymond, 2012; Raymond \& O'Brien, 2009). If effects were due to value, participants should report perceiving the figure on the side of the border where a portion of the low-probability loss stimulus is sketched (since this stimulus has lower predictiveness, but higher value). In contrast, if effects are due to predictiveness, participants should report perceiving the figure on the side of the border where a portion of the high-probability loss stimulus is suggested (since this stimulus has high probability, but lower value). Note that to ensure that participants had learned the reward contingencies, we analyzed test-phase data only for participants who met a criterion of $80 \%$ choices of the optimal stimulus in the learningphase.

The primary hypotheses, methods, and analysis protocols for this study were preregistered on the Open Science Framework (https://osf.io/ye8dw/). Nonpreregistered analyses are treated as exploratory.

\section{Experiment 1}

\section{Method}

\section{Participants and apparatus}

Pilot data (available on the Open Science Framework) were submitted to a $t$ test power analysis using $\mathrm{G}^{*}$ Power, using Cohen's $d$, which indicated that 18 participants would yield $80 \%$ power $(N=30$ for $90 \%$ power) to detect a significant 


\section{a) Stimuli presented during Learning}

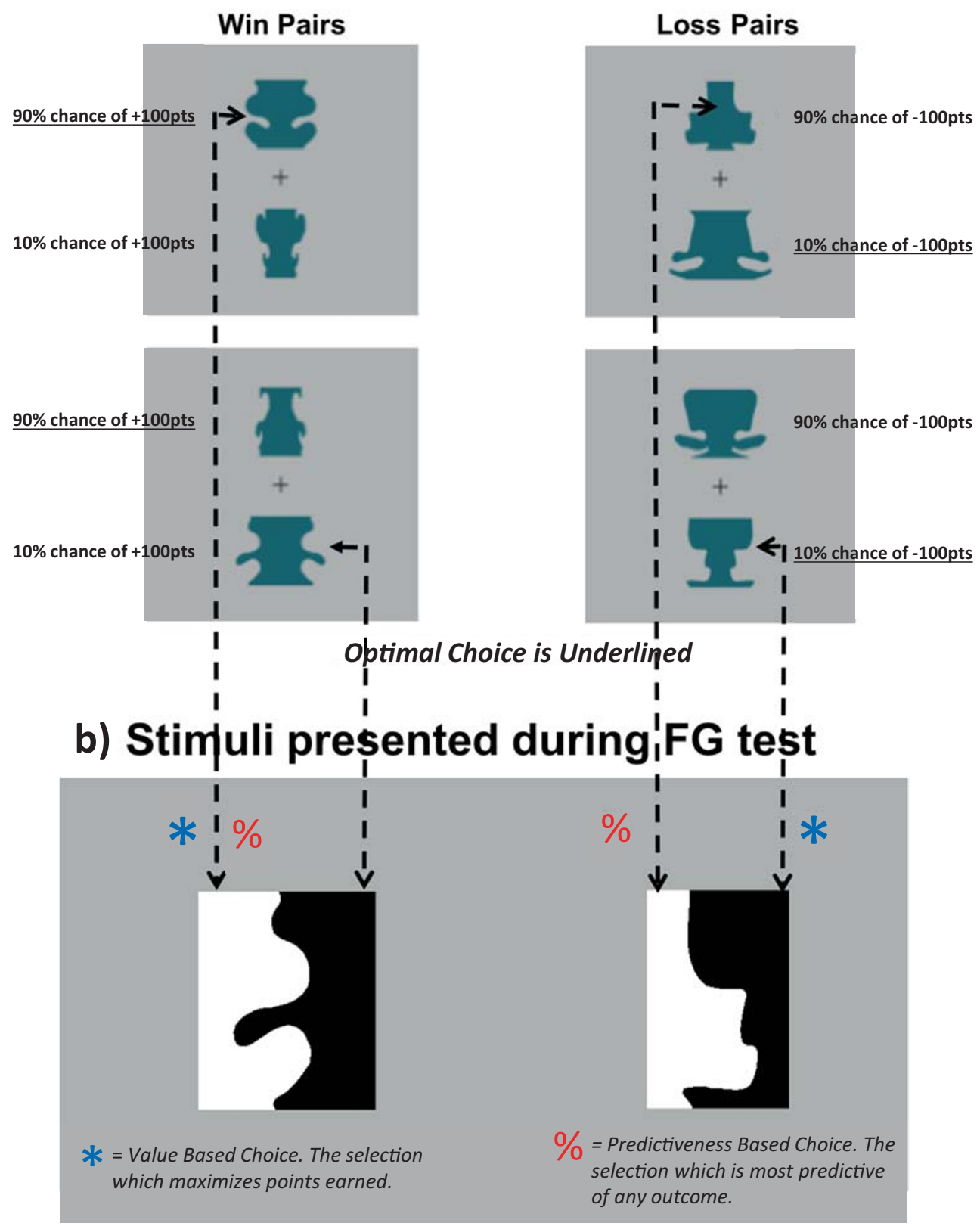

Fig. 2 a Design of the learning phase. On each trial, participants were presented with a pair of shapes (presented in either dark teal [as here] or light teal) and chose one. Depending on the pair presented, and their choice, they could then win or lose points. For "win pairs," one shape was associated with a $90 \%$ chance of winning 100 points (and a $10 \%$ chance of winning nothing), and the other with a $10 \%$ chance of winning 100 points (and 90\% chance of winning nothing). For "loss pairs," one shape was associated with a $90 \%$ chance of losing 100 points (and a $10 \%$ chance of losing nothing), and the other with a $10 \%$ chance of losing 100 points (and 90\% chance of losing nothing). The optimal choice on each trial is underlined in the figure. b Examples of stimuli presented in the

difference between proportion of trials selected as figure for win trials and loss trials. Previous eye-tracking studies investigating the impact of reward learning on attention have used figure-ground (FG) test phase. The border between the white and black regions of the stimulus presented on each trial traced the contour of two of the shapes that had been experienced in the preceding learning phase on opposite sides. For win-pair stimuli, the shape on one side was a $90 \%$-win stimulus, and the shape on the other side was a $10 \%$-win stimulus. For loss-pair stimuli, the shape on one side was a $90 \%$-loss stimulus and the shape on the other side was a $10 \%$-loss stimulus. Blue asterisks indicate a value-based choice (high-probability win and low-probability loss), and red percent sign indicate a predictiveness based choice (high-probability win and high-probability loss). (Color figure online)

approximately 30 participants (e.g., Le Pelley et al., 2015; Pearson, Donkin, Tran, Most, \& Le Pelley, 2015). Therefore, we aimed to collect useable data from 30 
participants, with a minimum sample size of 18 , depending on logistical constraints.

Fifty first-year psychology students $\left(M_{\mathrm{age}}=19.58\right.$ years, $S D_{\text {age }}=2.21$ years; 26 men, 24 women) participated for course credit and monetary rewards that were contingent on performance in the learning phase. Rewards ranged between AUD $\$ 8$ to AUD\$15. All research reported here was approved by the UNSW Sydney Human Research Ethics Advisory Panel (Psychology), and all participants gave informed consent.

Participants were tested in individual cubicles, each of which contained a Tobii TX300 eye tracker (sample rate 300 $\mathrm{Hz})$ mounted on a 23 -inch monitor $(1,920 \times 1,280$ resolution, $60-\mathrm{Hz}$ refresh rate). Head position was stabilized using a chin rest $\sim 60 \mathrm{~cm}$ from the screen. Stimulus presentation was controlled by MATLAB using Psychophysics Toolbox extensions (Brainard, 1997; Kleiner, Brainard, \& Pelli, 2007; Pelli, 1997). Auditory feedback was delivered over headphones, and participants made all responses using the keyboard. The eye tracker was calibrated for each participant at the start of the learning phase.

\section{Materials}

In the learning phase, eight shapes (each approximately $2^{\circ} \times$ $2^{\circ}$ visual angle) were clustered into four pairs, which were held constant across participants and throughout the phase. Two of the pairs were "win" pairs and two were "loss" pairs, with the win-loss associations balanced across participants. The shapes appeared on a medium-gray background (red, green, blue [RGB] values: $182,182,182$ ); on each trial during the learning phase, both shapes were presented in either dark teal (RGB: 16, 100, 112), which was darker than the background, or light teal (RGB: 41, 213, 199), which was lighter than the background. Each shape pair was presented in light and dark teal an equal number of times so that contrast polarity did not vary with either value or probability during the first phase. The two shapes in each pair were positioned with their centres $5^{\circ}$ above and below the screen centre.

The learning stimuli were designed so that the highprobability reward shape in one pair of win stimuli and the low-probability reward shape in the other pair of win stimuli would fit like jigsaw pieces if they abutted each other (see Fig. 2 ). In other words, the low-probability reward shape in one win pair was the shape sketched on the groundside of the border of the high-probability reward shape in the other win pair, and vice versa. The same was true of loss pairs. Participants were unaware of this relationship, which would have required comparing shapes across learning trials. There was no incentive to make this comparison, which in any case would be difficult: The learning stimuli were small in area, symmetric, closed, enclosed, and relevant to the task. Hence, the figures were highly likely to be perceived on the inside of the borders; the outsides then would have appeared to be shapeless grounds to the stimuli in the learning phase. The use of these stimuli in the learning phase allowed construction of figure-ground test stimuli that paired high-predictiveness reward (or loss) against low-probability reward (or loss).

In the figure-ground test phase, the stimulus on each trial was a rectangle $\left(3^{\circ} \times 2^{\circ}\right)$ presented in the centre of the screen on a gray (RGB: 182, 182, 182) background. This rectangle consisted of two equal-area regions - one white and one black - separated by a central articulated border (see examples in Fig. 2b). Critically, the central border of the rectangle on each trial traced the contour of two of the shapes that had been presented in the learning phase no opposite sides. For win test pairs, the articulated border sketched a portion of a high-probability win shape on one side and a portion of a low-probability win shape on the other side. For loss pairs, the central articulated border traced a portion of a high-probability loss shape on one side and a lowprobability loss shape on the other side. Note that for win trials, the high-probability and high-value side was the same, whereas for loss trials, high-probability and high-value sides were opposite one another. The borders from the learning phase were carried forward to the figure-ground test phase (see Fig. 2 for an illustration). Low-level features between the two regions such as convexity, symmetry, and surface area were matched in the figure-ground displays.

\section{Design and procedure}

Learning phase Full instructions given at the start of the learning phase can be found at the Open Science Framework (https://osf. io/ye8dw/). Briefly, participants were informed that they would be shown two shapes on each trial, and would have to choose one; that they could win or lose points depending on their choices; and that their aim should be to win as many points as possible since this would determine their monetary bonus at the end of the experiment (cash was visible on an adjacent table to render the reward salient). They were further told that there would be win pairs and loss pairs, for which they could win or lose 100 points (respectively) depending on their choice, and that choosing one of the shapes in each pair was much more likely to result in winning or losing points (respectively) than choosing the other. Finally, they were told that sometimes shapes would be presented in a dark color, and sometimes in a light color, but this was irrelevant to the optimal choice on each trial.

Each trial of the learning phase began with presentation of a central cross. Participants were required to fixate this cross: Once they had accumulated $100-\mathrm{ms}$ gaze dwell time within $1.5^{\circ}$ visual angle of the fixation cross, the screen blanked, and after $200 \mathrm{~ms}$, the pair of shapes was presented, one above and one below fixation (Fig. 2a shows examples). Participants chose the top or bottom shape using the up or down arrow key, respectively.

Two of the pairs of shapes were win pairs, for which choice of one shape was consistently associated with a high (90\%) 
probability of winning 100 points, and choice of the other shape gave a low (10\%) probability of winning 100 points. The other two shape pairs were loss pairs, for which one shape gave a high $(90 \%)$ probability of losing 100 points, and the other shape gave a low (10\%) probability of losing 100 points. On each trial, if participants did not win or lose 100 points, the outcome was neutral (they neither won nor lost points). Therefore, on each trial there was an optimal choice: For win pairs, the optimal choice was the high-probability win shape; for loss pairs, the optimal choice was the lowprobability loss shape.

Visual and auditory feedback was provided immediately after participants made their response on each trial. The message "WIN: 100 points" (in green), "LOSE: 100 points" (in red), or "NOTHING: 0 points" appeared, as appropriate. Win feedback was accompanied by a short chord on the major scale (a happy sound); loss feedback was accompanied by a buzzer sound. Visual feedback was displayed for 1,200 ms, then the screen then blanked for 1,400 ms before the next trial began.

Participants completed three learning blocks. The first block (40 trials) comprised trials with one win pair and one loss pair; the second block (40 trials) comprised trials with the two remaining pairs. In the third block (320 trials), trials with all four pairs were intermixed. Within each block, each pair of stimuli was presented on an equal number of trials in random order. The top/bottom locations in which the shapes of each pair were presented on each trial were counterbalanced across the trials in each block (so each shape appeared in the top location on exactly half of the presentations of that pair). Participants took a short break every 40 trials.

Figure-ground test phase Following the learning phase, participants were informed that their task would now change, and their performance would no longer affect their monetary reward. Participants were given brief information regarding figure-ground segregation (e.g., definitions of figure and ground), and were told that in the subsequent task they would be shown displays with a black and a white region separated by a central border, and would be required to indicate whether they saw the figure on the left or right side of the border.

Participants performed 64 figure-ground test trials over four blocks, with black and white composite test stimuli as shown in the bottom part of Fig. 2. Each figure-ground display was approximately $5 \times 5 \mathrm{~cm}$. On each trial, a fixation cross appeared, and participants were told to initiate the trial when they were sure they could maintain fixation and not blink, by pressing the space bar. The screen then cleared, and after $300 \mathrm{~ms}$ the figure-ground display appeared for 100 $\mathrm{ms}$, followed by a blank screen for $100 \mathrm{~ms}$, and then a speckled black and white backward pattern mask for $100 \mathrm{~ms}$, before a response prompt appeared (the message "Was the figure on the left or right side of the border" shown centrally). The figure-ground display appeared briefly to isolate the first perceived figure-ground segregation, and the mask served to prevent participants from relying on lingering impressions in iconic memory for their judgments. In previous research, 100ms display exposures have been sufficient to reveal robust effects on figure assignment of both familiarity and the Gestalt priors of symmetry and convexity (e.g., Gibson \& Peterson, 1994; Peterson \& Gibson, 1994a; Peterson \& Salvagio, 2008; Mojica \& Peterson, 2014). Participants made their (unspeeded) response using the left or right arrow keys.

There were four basic figure-ground test displays. Two of these were win displays, which combined a high-probability win shape (90\% chance of win) from one of the win pairs in the learning phase with a low-probability win shape $(10 \%$ chance of win) from the other win pair in the learning phase. The other two figure-ground test displays were loss displays, which combined a high-probability loss shape $(90 \%$ chance of loss) from one of the loss pairs in the learning phase with a low-probability loss shape (10\% chance of loss) from the other loss pair. Each of these four basic displays was experienced 16 times in the figure-ground test phase, for a total of 64 trials. For each type of basic display, presentations were balanced within-subjects for horizontal configuration (flipped horizontally) to eliminate effects due to left/right hemispheric preference, and for contrast polarity (i.e., which region was black, and hence lower in contrast than the medium-gray background, and which was white, and hence higher in contrast than the medium-gray background). This yielded four variants of each display type: horizontal configuration $(2) \times$ luminance (2). Each of these four variants of each display type appeared once per block of 16 trials, in random order, and there were four such blocks in the figure-ground test phase. Thus, the "optimal" region (see Fig. 2) appeared on both sides of the display, in both contrasts, an equal number of times.

\section{Results}

We conducted all analyses using a Bayesian approach which allows us to quantify evidence for the null (Onie, Notebaert, Clarke, \& Most, 2019). All analyses were conducted in JASP (Version 0.11.1) and JZS priors (Rouder \& Morey, 2012).

\section{Exclusions}

To ensure that participants whose data were entered in the analysis of figure-ground reports had learned the reward contingencies, we set an exclusion criterion based on responding during the learning phase: Participants had to choose the optimal shape in each of the four pairs on at least $80 \%$ of trials in the last $25 \%$ of trials. Based on this criterion, the data from 13 participants were excluded from subsequent analyses, leaving a sample of 37 participants $\left(M_{\text {age }}=19.5\right.$ years, $S D_{\text {age }}=2.22$ years, 21 men, 16 women). 


\section{Eye-gaze data}

We analyzed eye-tracking data from the learning phase to establish whether differences in predictiveness and/or value produced reliable changes in attention to stimuli (cf. Le Pelley et al., 2016) that might then persist to influence figure-ground segregation in the subsequent test phase. Specifically, we analyzed eye gaze from the last 200 trials of the learning phase - by this point, participants had had ample opportunity for learning, and mean choice of the optimal stimulus was high (>95\%), as seen in Table 1. A Bayesian $t$ test comparing percentage optimal choice over the last 200 trials revealed moderate evidence that there was no difference between win and loss trials $\left(t\right.$ test $\left.\mathrm{BF}_{01}=5.512\right)$.

The eye tracker sampled gaze location at $300 \mathrm{~Hz}$ (i.e., one sample every $3.33 \mathrm{~ms}$ ). Occasional failures to track (e.g., due to blinks) led to missing data for some samples (overall, $3.09 \%$ of samples were missing). We first used linear interpolation to fill gaps shorter than $75 \mathrm{~ms}$, and then applied smoothing using a moving-average window with a width of five samples $(16.7 \mathrm{~ms})$.

Recall that the shape stimuli presented in the learning phase appeared on the vertical midline, with their centers 200 pixels (5.1 $1^{\circ}$ visual angle) above and below the central fixation point. Hence, we were particularly interested in the vertical $(y)$ coordinate of gaze location. Figure 3 shows the mean $y$ coordinate of each participant's gaze location, averaged over the final 200 trials of the learning phase, for the first $1,000 \mathrm{~ms}$ after stimulus onset of each trial. Data are shown as if the optimal shape (highprobability win stimulus on win trials; low-probability loss stimulus on loss trials) were in the top position on every trial (if the optimal stimulus had actually appeared in the bottom position, the data for that trial were reflected along the horizontal midline of the screen). The gaze data revealed eye movements toward the optimal stimulus in both win and loss pairs, but these eye movements were generated earlier after stimulus onset for win pairs than for loss pairs: The point at which the mean $y$ coordinate of gaze first deviated from screen center (at $p$ $<.005$, uncorrected) was at $303 \mathrm{~ms}$ after stimulus onset on win trials and $427 \mathrm{~ms}$ after stimulus onset on loss trials. This is unlikely to be a consequence of a difference in strength of learning of the underlying associations, since our previous analyses suggested that choice performance was similar for both trial types over the final 200 trials that were analyzed here.

Table 1. Experiment 1 percentage optimal choice over the last 200 trials in the learning phase

\begin{tabular}{lll}
\hline Trial type & Percentage optimal choice & $S D$ \\
\hline Win trials & $97.361 \%$ & $8.445 \%$ \\
Loss trials & $97.611 \%$ & $3.147 \%$ \\
\hline
\end{tabular}

Note. Each trial type consists of two different pairs of stimuli
One interpretation of these findings is that participants were slower to start moving their eyes on loss trials than win trials. Another possibility is that trial type did not influence how long it took participants to initiate an eye movement, but did influence the likelihood that this first eye movement would be in the direction of the optimal stimulus. To investigate this question, we used a velocity-threshold identification (I-VT) algorithm (Salvucci \& Goldberg, 2000) with a velocity criterion of $40^{\circ}$ visual angle per second to detect saccades in the gaze data. For this analysis, we discarded trials in which no saccade was detected (4.42\% of all trials). The latency of the first saccade after stimulus onset did not differ significantly between win trials $(M$ $=266.8, S E M=9.3 \mathrm{~ms})$ and loss trials $(M=268.4, S E M=9.1$ $\mathrm{ms}$ ), $t<1$, with a Bayesian $t$ test (conducted using JASP with the default Cauchy prior) revealing moderate evidence in favor of the null $\left(B F_{01}=4.98\right)$. Figure 4 shows location data for starting points and subsequent fixations. These data suggest that, for win trials, the first saccade and fixation was reliably directed toward the optimal stimulus: The mean fixation was significantly different from centre in the direction of the optimal stimulus with a Bonferroni-adjusted $t$ test $(p<.001)$. In contrast, for loss trials, the first saccade did not reliably go toward the optimal stimulus; mean fixation did not differ significantly from screen center until second fixation onward. When directly comparing win and loss trials, fixation at win trials and loss trials differed significantly on the first fixation $(p<.001)$, second fixation $(p=0.014)$, and third fixation $(p=.028)$.

To summarize, the gaze data demonstrate that learning induced reliable changes in overt attention. More specificallyand consistent with previous research (see Le Pelley et al., 2016) - these data suggest that attention was influenced by learning about value and predictiveness. The effect of value is demonstrated in that attention typically went toward the optimal (high value) stimulus on each trial. Notably, this shift of overt attention toward the optimal stimulus occurred more rapidly on win trials than on loss trials, even when performance (in terms of response accuracy) was similar for both trial types. This finding is corroborated by the fixation analysis, where in win trials the first fixation was reliably biased toward the optimal stimulus, whereas in the loss trials only the second fixation was biased toward the optimal stimulus. This finding is consistent with the idea of competition between the effects of value and predictiveness on attention: On win trials, these properties are aligned (the optimal stimulus is the high-probability win shape), whereas on loss trials, they are in opposition (the optimal stimulus is the low-probability loss shape), and this competition may result in slower attentional selection of the optimal stimulus on loss trials.

\section{Figure-ground organization}

We calculated the mean proportion of trials from the figureground test phase on which participants perceived the figure on the side of the border where the optimal stimulus was sketched 

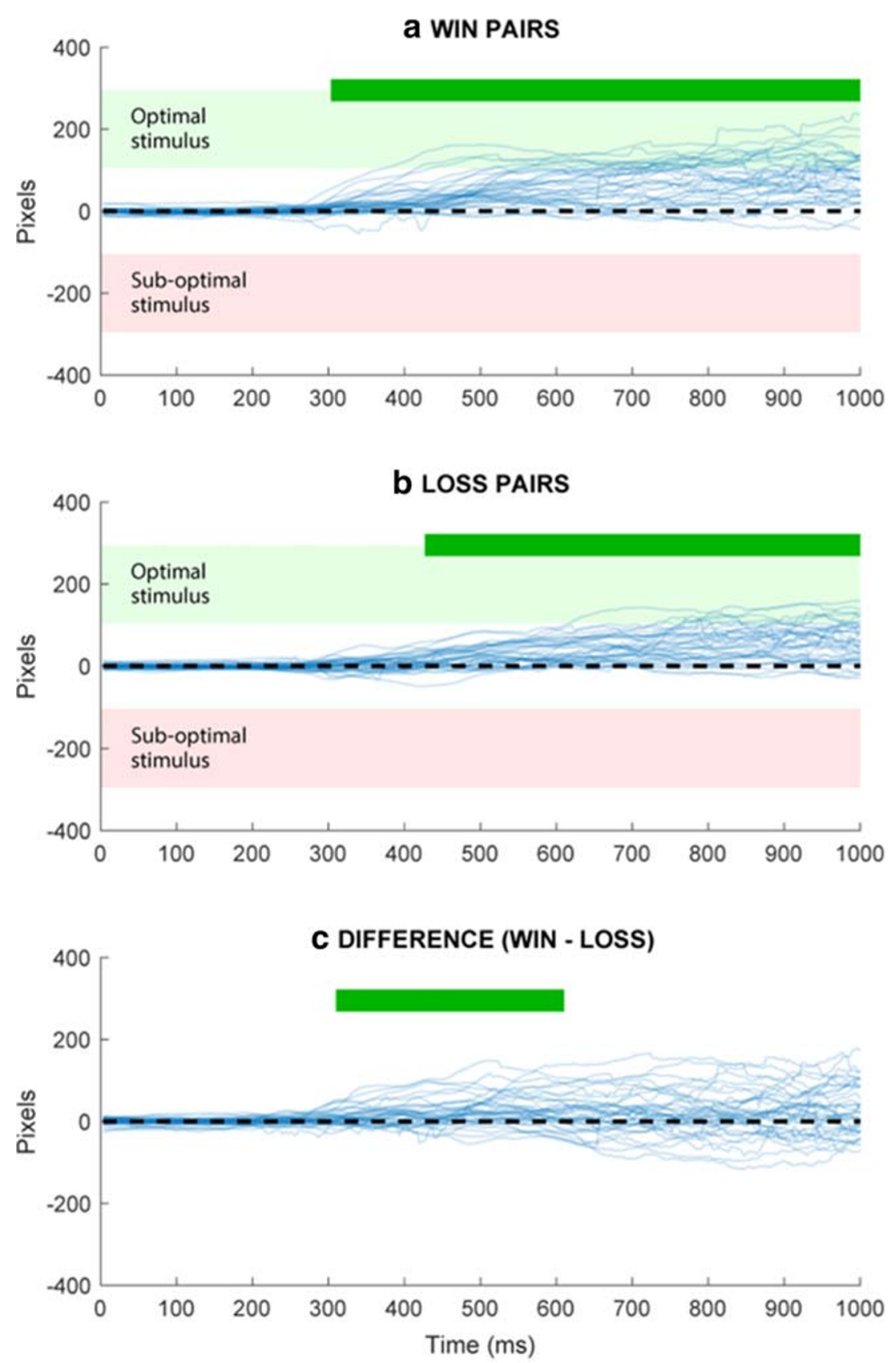

Fig. 3 Gaze data from the final 200 trials of the learning phase of Experiment 1. Data are time-locked to the onset of the stimulus display (i.e., time $=0$ shows the point at which the stimuli were presented). For illustration purposes, data are shown as if the optimal shape (highprobability win stimulus on win trials; low-probability loss stimulus on loss trials) were in the top position on every trial (if the optimal stimulus had actually appeared in the bottom position, the data for that trial were reflected along the horizontal midline of the screen). a Data for win trials. b Data for loss trials. Blue lines show the mean $y$ coordinate (i.e., vertical position), in pixels, of each participant's gaze over the first $1,000 \mathrm{~ms}$ of each trial. The dotted black line represents the center of the screen (initial

separately for trials featuring displays constructed from the stimuli presented on win trials versus loss trials during learning. We then used one-sample Bayesian $t$ tests against the proportion expected by chance $(0.5)$ to investigate whether prior learning fixation point); the light-green and light-red regions (the shaded regions above and below the 0 coordinate respectively) show. Green bars at the top of each panel show 3.33-ms samples for which a one-sample $t$ test indicated that mean vertical gaze-point deviated from screen center $(p<$ .005 , uncorrected): In all cases, this deviation was in the direction of the optimal stimulus. This analysis shows that gaze (overt attention) moved toward the optimal stimulus more rapidly for win pairs than for loss pairs. c Difference between win and loss trials for each participant, with green bar showing the period over which gaze differed between conditions at $p$ $<.005$ (paired-samples $t$ test). (Color figure online)

experience produced a systematic bias in figure-ground segregation.

For figure-ground displays constructed from the stimuli presented on win trials during learning, this analysis would 


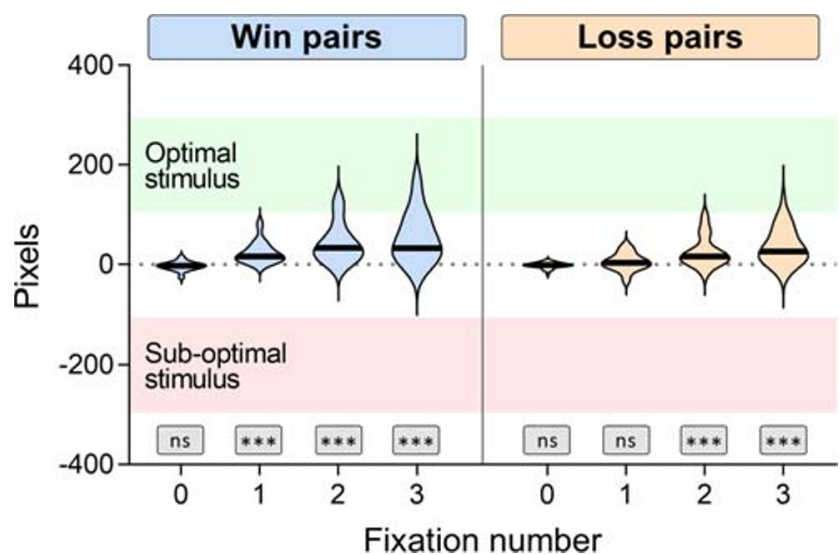

Fig. 4 Fixation analysis from the final 200 trials of the learning phase of Experiment 1, for win trials and loss trials. Plots show the $y$ coordinate (i.e., vertical position) in pixels of successive fixations following stimulus onset, where fixation number 0 corresponds to gaze location when stimuli appeared, fixation number 1 is the location of the next fixation and so on. The dotted black line represents the center of the screen (location of the central cross that participants were instructed to fixate before stimuli appeared). The light-green and light-red regions (shaded regions above and below the horizontal midline respectively) (data are plotted as if the optimal shape were in the top position on every trial). Violin plots are populated by the fixation points by all participants, while the black bars represent the mean fixation across all participants. Symbols above fixation number represent the outcome of a Bonferroni-corrected $t$ test ( $n s=$ nonsignificant, $* p<.05$, $* * p<.01, * * * p<.001$ ). This analysis shows that for win pairs, from the first fixation after stimulus onset, fixation was significantly different from the centre of the screen in the direction of the optimal stimulus. (Color figure online)

reveal whether the added effects of predictiveness and value had an impact on figure-ground segregation because both favored perceiving the figure on the same side of the test displays as the figure. The analysis revealed moderate evidence that this was not the case, however $(M=0.514, S D=$ $\left.0.126, \mathrm{BF}_{01}=4.640, \delta=0.098,95 \% \mathrm{CI}[-0.204,0.412]\right)$.

For loss trials, this analysis would reveal whether predictiveness or value separately had any impact on figureground segregation (as the high-value and high-probability shape competed for figural assignment). If value affects figure assignment, the figure should be perceived on the side of the central border where the optimal stimulus was sketched more often than chance. By contrast, if predictiveness affects figure assignment, the figure should be perceived on the side of the central border where the optimal stimulus was sketched less often than chance because the shape highly predictive of loss was suggested on the opposite side of the central border from the optimal shape. However, the analysis revealed moderate evidence that neither value nor predictiveness affected figural assignment $\left(M=0.506, S D=0.138, \mathrm{BF}_{01}=5.478, \delta=0.042,95 \%\right.$ CI $[-0.271,0.351])$.

We then directly compared whether there were differential effects of wins and losses on perceiving the optimal region as figure. We performed a paired-samples $t$ test between win and loss trials, using the proportion of times the optimal region was perceived as figure. The analysis revealed moderate evidence against any differential effects of win or loss on figure-ground segregation (win minus loss: $M=0.0076, S D=0.1043, \mathrm{BF}_{01}=5.159, \delta=0.087$, 95\% CI $[-0.336,0.510])$.

\section{Exploratory analyses}

One possibility is that the effect of reward learning on figureground organization was present, but fleeting. To examine this possibility, we constrained our analysis to the first block (16 trials: eight win, eight loss trials) of the figure-ground test phase. This more focused analysis still revealed evidence in favor of the null hypothesis, though this evidence was less conclusive than for the analyses of all test trials (win: $M=$ $0.537, S D=0.025, \mathrm{BF}_{01}=2.006, \delta=0.229,95 \% \mathrm{CI}$ [-0.079, 0.546]; loss: $M=0.537, S D=0.026, \mathrm{BF}_{01}=2.156$, $\delta=0.221,95 \%$ CI $[-0.096,0.534])$. Directly comparing trials featuring win and loss shapes using a paired samples $t$ test yielded moderate evidence against a differential effect of wins or losses on figure-ground segregation $\left(\mathrm{BF}_{01}=5.656, \delta=\right.$ $0.002,95 \%$ CI $[-0.412,0.426])$.

\section{Discussion}

In this study, we investigated the effect of reward learning on figure-ground segregation by training participants to associate rewards or losses to certain stimuli. The data suggest that participants learned the contingencies well, and any participants who did not meet criterion were removed. Learning the contingencies led to changes in overt attention during the learning phase, with a gaze bias evident toward optimal stimuli. Analysis of data from the figure-ground test phase suggested that reward learning did not affect figure-ground organization, however. One notable finding from the learning phase that may be relevant to the absence of effects of value or predictiveness on perceived figure assignment is that even when participants had learned the contingency, evidence of differential overt attention to the optimal versus suboptimal stimulus did not emerge until $>280 \mathrm{~ms}$ after stimulus onset (see Fig. 3a-b). Therefore, it could be that the 100-ms exposure of the figure-ground displays in the test phase was not long enough for differential attention (as a consequence of reward learning) to have an effect on figure-ground segregation. Notably, in this regard, in their study of the effects of attention, Vecera et al. (2004) presented figure-ground displays for $250 \mathrm{~ms}$. Therefore, in Experiment 2 we extended the exposure time of the figure-ground test displays to $300 \mathrm{~ms}$. Experiment 2 was documented on the Open Science Framework in a second preregistration (https://osf.io/ye8dw/). 


\section{Experiment 2}

\section{Method}

Thirty-one first-year psychology students $\left(M_{\mathrm{age}}=18.87\right.$, $S D_{\text {age }}=1.359$, men $=11$, women $=20$ ) participated for course credit, and performance-related monetary reward (typically AUD\$8-AUD\$15). With the exception that the figureground display was presented for $300 \mathrm{~ms}$ instead of $100 \mathrm{~ms}$, all other methodological details and data preparation procedures were identical to those of Experiment 1.

\section{Results}

\section{Exclusions}

Six participants were excluded for not selecting the optimal choice in $80 \%$ of the last quarter of the learning trials, leaving 25 participants $\left(M_{\text {age }}=19, S D_{\text {age }}=1.32\right.$, men $=11$, women $=$ 14).

\section{Eye-gaze data}

As in Experiment 1, we analyzed gaze from the last 200 trials of the learning phase-by this point, participants had had ample opportunity for learning, and mean choice of the optimal stimulus was high ( $>95 \%$ ), as seen in Table 2 . A Bayesian $t$ test comparing win and loss trials revealed inconclusive evidence as to whether there was a difference between win and loss trials $\left(\mathrm{BF}_{10}=2.494\right)$. Note, however, that percentage optimal choice in both types of trial was well above the $80 \%$ criterion.

Figure 5 shows mean $y$ coordinate of participants' gaze data locked to stimulus onset; for illustration purposes, data are shown as though the optimal stimulus had appeared in the top position on every trial. As in Experiment 1, eye movements were made to the optimal stimulus in both win and loss pairs, but these eye movements were generated earlier after stimulus onset for win pairs than for loss pairs: the point at which the mean $y$ coordinate of gaze first deviated from screen center (at $p<.005$, one-sample $t$ test) was at $330 \mathrm{~ms}$ after stimulus onset on win trials, and $453 \mathrm{~ms}$ on loss trials.

Table 2. Experiment 2 percentage optimal choice over the last 200 trials in the learning phase

\begin{tabular}{lll}
\hline Trial type & Percentage optimal choice & $S D$ \\
\hline Win trials & $97.500 \%$ & $3.063 \%$ \\
Loss trials & $95.808 \%$ & $4.000 \%$ \\
\hline
\end{tabular}

Note. Each trial type consists of two different pairs of stimuli
The latency of the first saccade after stimulus onset was determined (with $8.70 \%$ of trials excluded because no saccade was identified). As in Experiment 1, first saccade latency did not differ significantly between win trials $(M=308.9, S E M=$ $8.5 \mathrm{~ms})$ and loss trials $(M=243.8, S E M=16.5 \mathrm{~ms}), t<1$, with a Bayesian t-test revealing moderate evidence in favor of the null, $B F_{01}=3.36$. Figure 6 shows location data for starting points and subsequent fixations. These data suggest that, for both win and loss trials, the first saccade and fixation were reliably directed towards the optimal stimulus: the mean fixation was significantly different from centre in the direction of the optimal stimulus with a Bonferroni adjusted t-test. When directly comparing win and loss trials, fixation at win trials and loss trials do not differ at first fixation $(\mathrm{p}=.98)$ but did differ significantly at fixations $2(\mathrm{p}=.002)$ and $3(\mathrm{p}=.003)$.

In sum, both the stimulus and fixation data replicated the findings from Experiment 1: overt attention reliably moved to the optimal stimulus on each trial, but this shift occurred sooner on win trials than on loss trials. Hence, as in Experiment 1, both eye gaze and learning rate data distinguished win from loss pairs. Fewer trials were required to learn the optimal stimulus in win pairs than in loss pairs, and after the learning criterion had been reached the bias of the first fixation towards the optimal stimulus location did not differ significantly in win and loss trials; however, the degree of bias in fixation location differed significantly between win and loss trials for the second and third fixations.

\section{Figure-ground organization}

As in Experiment 1, we tested the hypothesis that participants would perceive the figure on the side where the border sketched a portion of the optimal stimulus by using a onesample $t$ test to compare their performance against chance level (0.5). The analysis revealed that there was moderate evidence that this was not the case for win trials $(M=0.509$, $S D=0.162, \mathrm{BF}_{01}=4.586, \delta=0.054,95 \%$ CI $[-0.317$, 0.415]). Likewise, the corresponding analysis for loss trials revealed anecdotal evidence in support of the null hypothesis $\left(M=0.530, S D=0.128, \mathrm{BF}_{01}=2.576, \delta=0.210,95 \% \mathrm{CI}\right.$ $[-0.171,0.593])$. Therefore, the results suggest that neither value when combined with predictiveness, nor value when placed in competition with predictiveness affected figureground segregation.

We then investigated whether there was a differential effect of wins and losses by directly comparing proportion of responses where figure status was assigned to the optimal shapes in the test phase using a paired-samples $t$ test, thus testing the effect of predictiveness. The analysis yielded moderate evidence suggesting no differential effect of wins or losses on figure-ground segregation $\left(\mathrm{BF}_{01}=3.594, \delta=\right.$ $0.181,95 \%$ CI $[-0.699,0.313])$. 

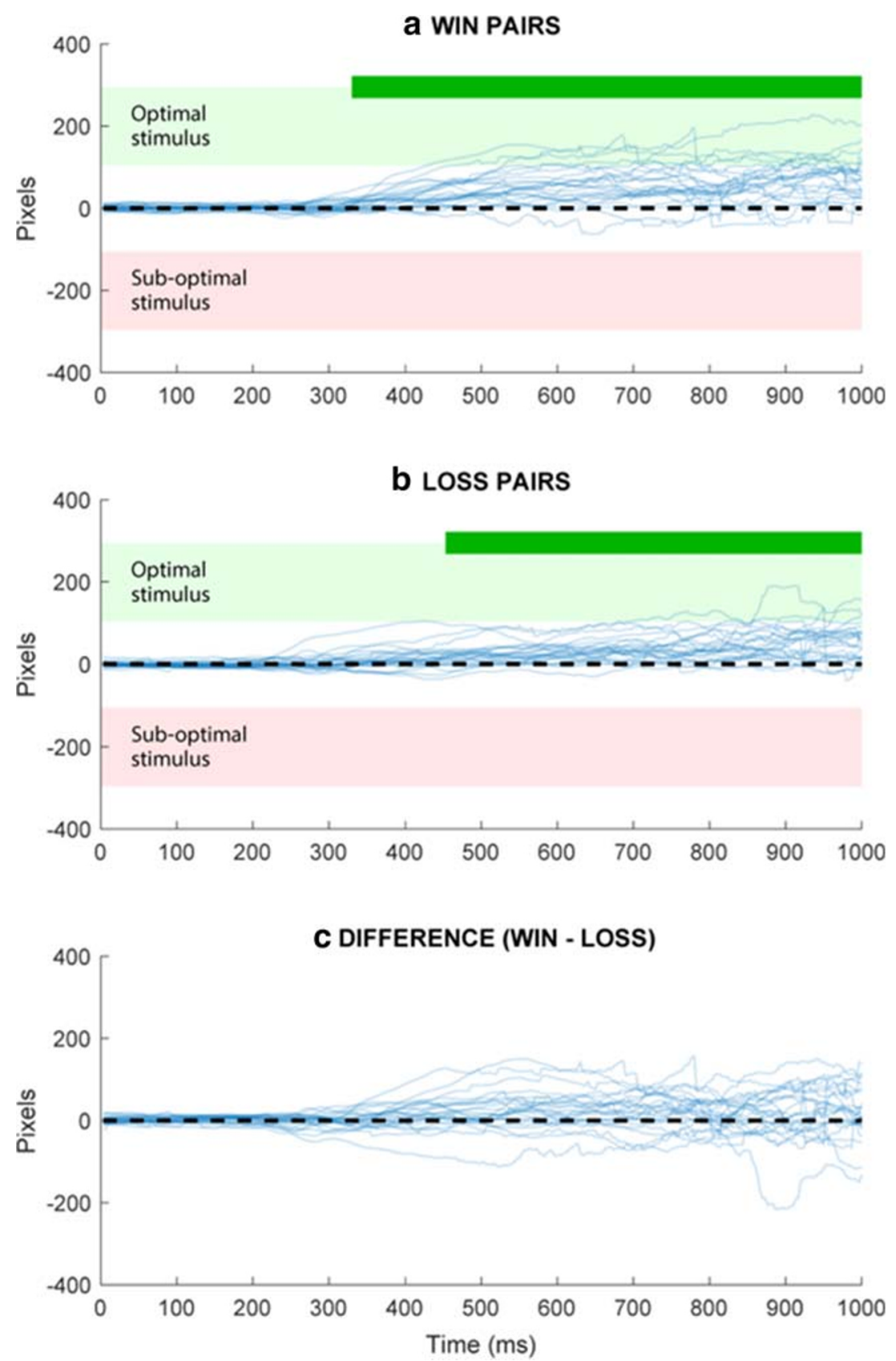

Fig. 5 Gaze data from the final 200 trials of the learning phase of Experiment 1. Data are time-locked to the onset of the stimulus display (i.e., time $=0$ shows the point at which the stimuli were presented). For illustration purposes, data are shown as if the optimal shape (highprobability win stimulus on win trials; low-probability loss stimulus on loss trials) were in the top position on every trial (if the optimal stimulus had actually appeared in the bottom position, the data for that trial were reflected along the horizontal midline of the screen). a Data for win trials. b Data for loss trials. c Differences (win minus loss). For further details, see the caption for Fig. 3

\section{Exploratory analyses}

To test whether the failure to find effects of value and predictiveness could be due to such effects being short lived, we restricted our analysis to the first block of trials in the figure-ground test phase. We performed one-sample $t$ tests for optimal choice on both win and loss trials, testing against chance level. The analysis revealed moderate evidence against any effect of value on figure-ground segregation (win: $M=$ $0.490, S D=0.180, \mathrm{BF}_{01}=4.578, \delta=-0.052,95 \% \mathrm{CI}$ [-0.428, 0.323]; loss: $M=0.510, S D=0.157, \mathrm{BF}_{01}=4.528$, $\delta=0.054,95 \%$ CI $[-0.309,0.425])$.

We then directly compared wins and losses using a pairedsamples $t$ test to investigate the differential effects of reward type on figure-ground segregation. The analysis revealed moderate evidence of no differential effects of reward type 


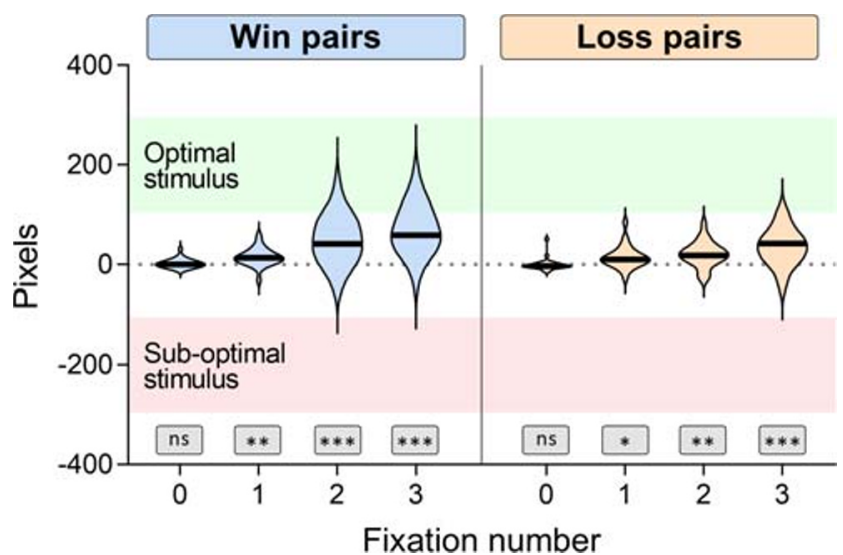

Fig. 6 Fixation analysis from the final 200 trials of the learning phase of Experiment 2, for win trials and loss trials. Details are as for Fig. 4

on figure-ground segregation $\left(\mathrm{BF}_{01}=4.214, \delta=-0.116,95 \%\right.$ CI $[-0.616,0.376])$.

Taken together, these results suggest that our failure to find an effect of reward learning on figure-ground segregation in Experiment 2 was not a consequence of this effect being fleeting.

\section{General discussion}

In this study, we investigated whether figure-ground perception is influenced by reward history, implementing reward learning while measuring gaze to index overt attention. In Experiment 1, the evidence suggested a differential effect of training with gains versus losses on learning and attention during the learning phase. The gaze data are consistent with findings reviewed by Le Pelley et al. (2016), indicating that learned value and learned predictiveness both modulate deliberate attentional focus, in that predictors of high-value outcomes receive higher levels of attention than predictors of low-value outcomes and predictors of high-probability outcomes receive higher levels of attention than predictors of low-probability outcomes.

Fixation analyses in Experiment 1 found that in the last 200 trials, when participants had learned the contingencies, mean fixation location on win trials differed from that on loss trials, with greater evidence of bias toward the optimal stimulus location in win trials. This pattern was present from the first to third fixation after stimulus onset. Further, while the first fixation location was significantly biased toward the optimal stimulus (versus the suboptimal stimulus) on win trials, on loss trials no significant bias was observed until the second fixation. Findings of Experiment 2 differed slightly: Here, we found that first fixations were significantly biased in both win and loss trials, with no significant difference between the two. However, we did find a significant difference between win and loss trials for the second and third fixations, with greater bias in win trials. Overall, we found evidence that even though participants had learned the contingency and there was evidence that choice data did not differ, there was still a differential effect of win and loss trials on overt attention. This took the form of either quicker orienting to the optimal stimulus on win trials (in Experiment 1) or fixations closer to the optimal stimulus in the second and third fixation (in Experiment 2).

One potential explanation for the reduced orienting on loss trials relative to win trials is that predictiveness and value competed to drive attention on loss trials (in which one shape had high probability but low value, and the other had low probability and high value). Specifically, covert attention might first be drawn to the high-probability stimulus, but must be then overcome to obtain the high-value reward. This contrasts with win trials, where the optimal choice was high in both probability and value. Competition takes time (e.g., Brooks \& Palmer, 2011; Peterson \& Enns, 2005; Peterson \& Lampignano, 2003). Hence, competition between allocating attention toward the high-probability versus high-value stimulus may be responsible for the slowed response, even though participants fully learned the contingency on the loss trials.

Despite evidence that participants had learned the reward contingencies and that attention was drawn to these stimuli during the learning phase, neither value nor predictiveness had an influence on figure-ground organization during the test phase of Experiment 1 (in which figure-ground displays were presented for $100 \mathrm{~ms}$ ). In Experiment 2, we increased the exposure time of the figure-ground display to $300 \mathrm{~ms}$ to probe the possibility that the test display had been exposed too briefly for effects of reward history to emerge in Experiment 1 . Nevertheless, Experiment 2 replicated the findings of Experiment 1: we observed effects of value and predictiveness on learning and attention during the learning phase, but no evidence for an effect on figure-ground segregation in the subsequent test phase.

Thus, the two experiments reported here failed to show evidence that value (or predictiveness) influences figure assignment, even though, unlike the previous experiments investigating whether reward affects figure assignment, our data showed strongly that participants had learned the reward contingencies and that their attention was biased toward the optimal stimulus during the learning phase of the experiment that ended just before the test phase.

Is it possible that the black and white figure-ground test displays were exposed too briefly for value to have an effect? The test displays were exposed for only $100 \mathrm{~ms}$ in Experiment 1, but for $300 \mathrm{~ms}$ in Experiment 2, which is more than enough time for past experience to affect figure assignment; such effects have been observed in masked exposures ranging from $57 \mathrm{~ms}$ to $100 \mathrm{~ms}$ (Gibson \& Peterson, 1994; Peterson \& Gibson, 1994a). Nevertheless, we observed no effects of learned value on figure assignment. We did not record eye movements while participants viewed the test displays, but 
since evidence suggests that both eye fixation on and attention to regions of figure-ground displays are figural priors, our results suggest that the optimal stimuli did not attract attention and/or eye gaze before figure assignment. Although earlier work by Smith and Hochberg (1954) determined that $333 \mathrm{~ms}$ was sufficient for half of their subjects to perceive both alternatives in their test displays, and therefore to caution against interpreting positive effects of value found with such long displays, this is not a concern here because we failed to observe effects of value/predictiveness on figure assignment.

One important difference between our test displays and the learning displays is that during the learning phase, myriad Gestalt priors favored the assignment of figural status to the optimal/predictive shapes (e.g., symmetry, closure, small area), whereas Gestalt priors were equated for the two regions in the test displays such that effects of value and/or predictiveness, if present, could be observed. During the learning phase, the optimal stimulus attracted attention/gaze, consistent with what has been shown in many laboratories using various methods. Nevertheless, we found no evidence that the optimal stimulus is more likely to be perceived as figure when no other priors favored that side as the figure in the black and white figure-ground test displays. Hence, our experiments join with the previous evidence to suggest that learned value does not affect figure assignment, even though we used a larger sample of subjects and required participants to meet a learning criterion.

There are several reasons we and others (e.g., Rock \& Fleck, 1950; Smith \& Hochberg, 1954) may have failed to find effects of value on figure assignment. First, perhaps not all forms of experience can influence figure assignment: our results suggest that even though shape familiarity affects figure assignment, learned value does not.

Second, perhaps value-based attention operates only after perceptual organization processes have segregated the visual field into figures and grounds. None of the previous experiments investigating effects of value on attention can rule out this possibility.

Third, it may be that in our attempt to be certain participants had learned the reward contingencies, we presented the stimuli too many times during learning (100 times each), such that based on past experience alone, the competition between the two potential shapes on opposite sides of the borders in the figure-ground test stimuli was too high to allow observation of effects of value. One form of experience (previous exposure to the shapes that were equal for high-value and low-value stimuli) may have swamped effects of another form of past experience-learned value. Previous experimenters (e.g., Rock \& Fleck, 1950; Schafer \& Murphy, 1943; Smith \& Hochberg, 1954) had not presented their stimuli as often as we did, but they did present each stimulus at least 15 times. Perhaps effects of value could be seen if fewer training trials were used (although this balance may be difficult to achieve while ensuring enough exposure to produce value learning).

Fourth, although the portions of the learned stimuli that were present in the figure-ground test displays were insufficient to attract attention and/or to produce effects of value on figure assignment, such effects might have been evident had the whole optimal stimulus been present in the figure-ground test displays. In many previous experiments, portions of wellknown objects have been sufficient to reveal effects of past experience on figure assignment in test display like those we used here (e.g., Gibson \& Peterson, 1994; Peterson \& Gibson, 1994a, 1994b; Peterson et al., 1991) and to reveal via indirect measures that shape memories were activated during figure assignment (Cacciamani, Mojica, Sanguinetti, \& Peterson, 2014; Peterson \& Skow, 2008; Sanguinetti, Allen, \& Peterson, 2014). Therefore, we adapted the method of presenting only a portion of the critical stimulus in the current experiments. In previous studies demonstrating the effects of familiarity on figure-ground assessment, it is likely that those wellknown objects had previously been encountered both when they were partially occluded as well as when they were not occluded; hence, participants probably had experience recognizing them based on a portion of the object as well as the whole object. Perhaps previous experience with occluded objects is necessary to observe effects of experience on figure assignment with only a portion of a familiar object. Future experiments can test whether effects of the learned value of stimuli on figure-ground perception are evident if the optimal stimulus occasionally appeared under conditions of occlusion during the learning phase.

Fifth, rewards were suspended during the figure-ground test phase, and participants were informed of this. We suspended rewards so participants would report their first perceived figure-ground organization and not change their reports in pursuit of higher rewards. If this suspension of reward could be eliminated, perhaps the influence of reward contingencies on figure assignment would be evident (i.e., with rewards in effect during the figure-ground test period). That said, many prior studies have demonstrated persistent effects of differences in predictiveness and value on attention even when predictiveness is subsequently equated (e.g., Le Pelley, Beesley, \& Griffiths, 2011; Le Pelley \& McLaren, 2003; Le Pelley et al., 2013) or rewards are suspended (e.g., Anderson et al., 2011; Le Pelley, Watson, Pearson, Abeywickrama, \& Most, 2019; Watson et al., 2019).

Sixth, effects of reward on figure-ground segregation may exist, but observation of such effects might require participants to consciously recognize the objects from the training phase in the test phase, at least when they were perceived as figure. While it was not documented, postexperiment debriefing revealed that almost no participants realized the connection between the objects in the training phase and the region contours in the figure-ground phase. 
Seventh, there is the possibility that the effects of reward and attention were fleeting and had dissipated by the figureground test phase. Previous evidence suggests that this is unlikely: Existing studies using reward manipulations similar to ours (and operating over similar time scales) have shown clear influences on performance in subsequent object-recognition tasks (e.g., Failing \& Theeuwes, 2015; O'Brien \& Raymond, 2012; Raymond \& O'Brien, 2009). These findings suggest that the type of reward-learning task used here can produce effects that persist and modulate behavior beyond the training period. That said, we did not explicitly test whether the effects of learning persisted in our study, and so this remains an open issue. Future studies may be able to test whether the effects of reward persisted with additional reward trials after the figure-ground phase.

A strength of our current design is that it enables observation of the competition between value and predictiveness on loss trials. Had a summed impact of predictiveness and value emerged when "win" regions were pitted against each other (a condition in which predictiveness and value were conflated), the pattern of figure-ground assignment when "loss" regions were pitted against each other would have enabled disentanglement of which aspect was instrumental. In the end, no effect of either predictiveness or value (or both combined) emerged when either "win" or "loss" regions were pitted against each other. Is it possible that predictiveness and value were in fact both exerting an influence on figure assignment on loss trials, but (since these effects were operating in opposing directions, favoring opposite sides of the test stimuli as figures) they cancelled one another out? We do not consider this explanation viable because on win trials, when both factors favored the same side as figure (i.e., they should have cooperated rather than competed), we observed no effects of learning history on figure assignment.

On this issue, it is notable that our design for examining (and potentially disentangling) effects of wins and losses differed somewhat from that used in previous studies. In the figure-ground test phase of previous studies (Schafer \& Murphy, 1943), win-associated regions were pitted against loss-associated regions, whereas in the current study both regions were either win associated or loss associated, differing only in the probability with which they predicted the respective outcome. Consequently, previous studies did not hold the potential to disentangle roles of value and predictiveness, whereas the current design did. Despite this potential strength of the current design, we did not find an effect of either value or predictiveness on figure-ground assignment in this study. In follow-up work, it may be fruitful to combine the eyetracking approach of the current study with the gain-versusloss aspect of earlier studies to further investigate any impact of value on figure-ground assignment.

\section{Attention and value}

In our study, eye-gaze data showed that the high-value (optimal) object reliably attracted attention during the learning phase of the experiment, especially on win trials, where both value and outcome probability were high for the optimal stimulus. Despite this, the shapes that reliably attracted attention in the learning phase were not perceived as figure more often during test. If we assume that a portion of the learned shape was sufficient to attract attention on the figure-ground test trials in the figure-ground test phase (as the whole shape did in the learning phase), these findings are inconsistent with previous reports that attention influences figure-ground organization (e.g., Vecera et al., 2004; though we note the possibility that the regions in the figure-ground test phase did not capture attention despite having the same contours as regions in the learning phase). We next review those previous studies and consider the strength of the evidence for the claim that attention influences figure-ground perception.

To investigate whether exogenous attention affected figure-ground perception, Vecera et al. (2004) used as study displays stimuli in which an articulated central border separated two equal-area regions of a study display; both regions were novel in shape, and no Gestalt priors favored one region as figure. They showed participants a study display for 100 $\mathrm{ms}$; a probe then appeared briefly on one of the regions, and then the study display was exposed alone for another $100 \mathrm{~ms}$. The test display then appeared: Participants were required to report which of two shapes had the same border as in the study display. Vecera et al. found that test trial performance was faster and more accurate when the border was repeated as a boundary of a shape on the side where the probe appeared in the study display rather than the opposite side. They took these data as evidence that the brief exposure of the probe had exogenously captured attention to that region, causing it to be perceived as the figure. While this is one potential interpretation, it is also possible that participants intentionally perceived the region on which the probe appeared as the figure (a postprobe strategy). They may have done this because, as Driver and Baylis (1996) showed, it is not possible to remember the shape of the border, per se. Remembering the shape of one figure would allow them to respond quickly on test trials when the border was repeated as a boundary of a shape on that side. When the test trials faced in the opposite direction, they could simply reverse the figure-ground organization of the test displays as Driver and Baylis' participants presumably did. This alternative interpretation raises questions regarding whether attention drawn to a region by the probe onset influenced perceived figure assignment or whether participants adopted a strategy of using the probe location in service of performing their task accurately (cf. Peterson \& Kimchi, 1994). Additional questions about the relationship between attention and figure assignment were raised by Peterson, 
Mojica, Salvagio, and Kimchi (2017), who found that attention allocated toward one region of a display in search of a target did not influence perceived figure-ground organization. Thus, if attentional allocation in and of itself has little or no influence on figure assignment, then perhaps it is not surprising that we found no effect of value on figure assignment, even though value affects attentional allocation.

In contrast to our findings, there is evidence that reward alters binocular rivalry (e.g., Alpers, Ruhleder, Walz, Mühlberger, \& Pauli, 2005; Wilbertz, van Slooten, \& Sterzer, 2014). In those experiments, the effect of value on rivalry was probably mediated by attention to stimulus features present when reward was delivered (e.g., texture or color) rather than by shape, and these effects operated during the periods when reward was delivered. The present experiments did not examine the role of color. This discrepancy between findings may suggest that some types of competition such as interocular competition that underlies binocular rivalry may be open to effects of reward, whereas the type of competition underlies figure-ground assignment is not.

\section{Conclusion}

In conclusion, we conducted two experiments to investigate whether reward learning influences figure assignment. In both experiments, we demonstrated that participants had learned the reward contingencies relating to the different stimuli, and that, following learning, the optimal stimulus attracted attention. In the current study, such attentional allocation was overtly observable, in the form of eye movements. Yet both experiments yielded evidence that prior reward learning does not influence figure-ground organization. In the pantheon of factors that have been shown to affect figure-ground segmentation, reward learning, value, and predictiveness appear to have no place.

Acknowledgements and funding information This work was supported by Australian Research Council Discovery Project DP170101715 to M.L.P. and S.B.M., Australian Research Council Future Fellowship FT120110707 to S.B.M., and Office of Naval Research Grant N00014-14-1-067 to M.A.P. All authors were responsible for conceptualization, S.O. and M.L.P. were responsible for formal analysis, M.L.P. was responsible for programming and visualization. All authors contributed to writing of this manuscript (S.O.- original draft; M.A.P., M.L.P., \& S.B.M.- review \& editing). Michelle Burrola, Danielle Mathieson, and Jane Raymond, in collaboration with Mary Peterson presented a poster at VSS (Vision Sciences Society Conference) 2015 reporting two experiments that served as pilot work for the current experiments. We appreciate their work as a forerunner to the present experiments.

\section{References}

Alpers, G. W., Ruhleder, M., Walz, N., Mühlberger, A., \& Pauli, P. (2005). Binocular rivalry between emotional and neutral stimuli: A validation using fear conditioning and EEG. International Journal of Psychophysiology, 57(1), 25-32.

Anderson, B. A., Laurent, P. A., \& Yantis, S. (2011). Learned value magnifies salience-based attentional capture. PLOS ONE, 6, e27926. https://doi.org/10.1371/journal.pone.0027926c

Bahnsen, P. (1928). Eine Untersuchung über Symmetrie und Asymmetrie bei visuellen Wahrnehmungen [An investigation into symmetry and asymmetry in visual perception]. Zeitschrift für Psychologie, cviii, $129-154$.

Baylis, G. C., \& Driver, J. (1995). One-sided edge assignment in vision: 1. Figure-ground segmentation and attention to objects. Current Directions in Psychological Science, 4, 140-146.

Beesley, T., \& Le Pelley, M. E. (2010). The effect of predictive history on the learning of sub-sequence contingencies. The Quarterly Journal of Experimental Psychology, 63, 108-135.

Bonardi, C., Graham, S., Hall, G., \& Mitchell, C. (2005). Acquired distinctiveness and equivalence in human discrimination learning: Evidence for an attentional process. Psychonomic Bulletin \& Review, 12, 88-92.

Brainard, D. H. (1997) The Psychophysics Toolbox. Spatial Vision, 10, 433-436.

Brooks, J. L., \& Palmer, S. E. (2011). Cue competition affects temporal dynamics of edge assignment in human visual cortex. Journal of Cognitive Neuroscience, 23(3), 631-644.

Cacciamani, L., Mojica, A. J., Sanguinetti, J. L., \& Peterson, M. A. (2014). Semantic access occurs outside of awareness for the groundside of a figure. Attention, Perception, \& Psychophysics, 76(8), 2531-2547. https://doi.org/10.3758/s13414-014-0743-y

Chelazzi, L., Peralto, A., Santandrea, E., \& Libera, C. D. (2013). Rewards teach visual selective attention. Vision Research, 85, 58-72.

Deubel, H., \& Schneider, W. X. (1996). Saccade target selection and object recognition: Evidence for a common attentional mechanism. Vision Research, 36, 1827-1837.

Driver, J., \& Baylis, G. C. (1996). Edge-assignment and figure-ground segmentation in short-term visual matching. Cognitive Psychology, $31,248-306$

Failing, F. M., \& Theeuwes, J. (2015). Nonspatial attentional capture by previously rewarded scene semantics. Visual Cognition, 23, 82104. https://doi.org/10.1080/13506285.2014.990546

Gibson, B. S., \& Peterson, M. A. (1994). Does orientation-independent object recognition precede orientation-dependent recognition? Evidence from a cuing paradigm. Journal of Experimental Psychology: Human Perception \& Performance, 20, 299-316.

Goldreich, D., \& Peterson, M. A. (2012). A Bayesian observer replicates convexity context effects in figure-ground perception. Seeing and Perceiving, 25(3-4), 365-395. https://doi.org/10.1163/ 187847612 X634445

Griffiths, O., \& Le Pelley, M.E. (2009). Attentional changes in blocking are not a consequence of lateral inhibition. Learning \& Behavior, $37,27-41$.

Hendrikse, J. J., Cachia, R. L., Kothe, E. J., McPhie, S., Skouteris, H., \& Hayden, M. J. (2015). Attentional biases for food cues in overweight and individuals with obesity: A systematic review of the literature. Obesity Reviews, 16(5), 424-432. https://doi.org/10.1111/obr. 12265

Hoffman, J. E., \& Subramaniam, B. (1995). The role of visual attention in saccadic eye movements. Perception \& Psychophysics, 57, 787795. https://doi.org/10.3758/BF03206794

Hoffman, D. D., \& Singh, M. (1997). Salience of visual parts. Cognition, 63, 29-78. 
Jacobs, D. W. (1996). Robust and efficient detection of salient convex groups. IEEE Transactions on Pattern Analysis and Machine Intelligence, 18(1), 23-37.

Kahnt, T., Park, S. Q., Haynes, J. D., \& Tobler, P. N. (2014). Disentangling neural representations of value and salience in the human brain. Proceedings of the National Academy of Sciences of the United States of America, 111, 5000-5005.

Kanizsa, G., \& Gerbino, W. (1976). Convexity and symmetry in figureground organization. In M. Henle (Ed.), Vision and artifact. New York, NY: Springer.

Kleiner, M., Brainard, D., \& Pelli, D. (2007). What's new in Psychtoolbox-3? Perception, 36, ECVP Abstract Supplement.

Koffka, K. (1935). Principles of Gestalt psychology. Oxford, England: Harcourt, Brace.

Kowler, E., Anderson, E., Dosher, B., \& Blaser, E. (1995). The role of attention in the programming of saccades. Vision Research, 35(13), 1897-916.

Le Pelley, M. E., Beesley, T., \& Griffiths, O. (2011). Overt attention and predictiveness in human associative learning. Journal of Experimental Psychology: Animal Behavior Processes, 37, 220 229.

Le Pelley, M. E., \& McLaren, I. P. L. (2003). Learned associability and associative change in human causal learning. Quarterly Journal of Experimental Psychology, 56B, 68-79.

Le Pelley, M. E., Mitchell, C. J., Beesley, T., George, D. N., \& Wills, A. J. (2016). Attention and associative learning in humans: An integrative review. Psychological Bulletin, 142(10), 1111-1140. https:// doi.org/10.1037/bul0000064

Le Pelley, M. E., Pearson, D., Griffiths, O., \& Beesley, T. (2015). When goals conflict with values: Counterproductive attentional and oculomotor capture by reward-related stimuli. Journal of Experimental Psychology: General, 144, 158-171. https://doi.org/10.1037/ xge0000037

Le Pelley, M. E., Seabrooke, T., Kennedy, B. L., Pearson, D., \& Most, S. B. (2017). Miss it and miss out: Counterproductive nonspatial attentional capture by task-irrelevant, value-related stimuli. Attention, Perception, \& Psychophysics, 79, 1628-1642.

Le Pelley, M. E., Vadillo, M. A., \& Luque, D. (2013). Learned predictiveness influences rapid attentional capture: Evidence from the dot probe task. Journal of Experimental Psychology: Learning, Memory, and Cognition, 39, 1888-1900.

Le Pelley, M. E., Watson, P., Pearson, D., Abeywickrama, R. S., \& Most, S. B. (2019). Winners and losers: Reward and punishment produce biases in temporal selection. Journal of Experimental Psychology: Learning, Memory, and Cognition, 45, 822-833.

Lee, J., \& Shomstein, S. (2014). Reward-based transfer from bottom-up to top-down search tasks. Psychological Science, 25, 466. doi: https://doi.org/10.1177/0956797613509284

Livesey, E. J., Harris, I. M., \& Harris, J. A. (2009). Attentional changes during implicit learning: Signal validity protects a target stimulus from the attentional blink. Journal of Experimental Psychology: Learning Memory \& Cognition, 35, 408-422.

Livio, M. (2005). The equation that couldn't be solved. New York, NY: Simon \& Schuster Trade.

Luque, D., Morís, J., Rushby, J. A., \& Le Pelley, M. E. (2015). Goaldirected EEG activity evoked by discriminative stimuli in reinforcement learning. Psychophysiology, 52, 238-248.

Mojica, A. J., \& Peterson, M. A. (2014). Display-wide influences on figure-ground perception: The case of symmetry. Attention, Perception, \& Psychophysics, 76, 1069-1084. https://doi.org/10. 3758/s13414-014-0646-y

Navon, D. (2010). The effect of recognizability on figure-ground processing: Does it affect parsing or only figure selection? The Quarterly Journal of Experimental Psychology, 64(3), 608-624.

O’Brien, J. L., \& Raymond, J. E. (2012). Learned predictiveness speeds visual processing. Psychological Science, 23, 359-363.
Onie, S., Notebaert, L., Clarke, P., \& Most, S. B. (2019) Investigating the effects of inhibition training on attentional bias change: A simple Bayesian approach. Frontiers in Psychology https://doi.org/10. 3389/fpsyg.2018.02782

Pearson, D., Donkin, C., Tran, S. C., Most, S. B., \& Le Pelley, M. E. (2015). Cognitive control and counterproductive oculomotor capture by reward-related stimuli. Visual Cognition, 23(1/2), 41-66. https://doi.org/10.1080/13506285.2014.994252

Pelli, D. G. (1997) The VideoToolbox software for visual psychophysics: Transforming numbers into movies, Spatial Vision, 10, 437-442.

Pessoa, L. (2015). Multiple influences of reward on perception and attention. Visual Cognition, 23(1/2), 272-290. https://doi.org/10.1080/ 13506285.2014.974729

Peterson, M. A. (1994). Object recognition processes can and do operate before figure-ground organization. Current Directions in Psychological Science, 3, 105-111.

Peterson, M. A., \& Enns, J. T. (2005). The edge complex: Implicit perceptual memory for cross-edge competition leading to figure assignment. Perception \& Psychophysics, 4, 727-740.

Peterson, M. A., \& Gibson, B. S. (1994a). Must figure-ground organization precede object recognition? An assumption in peril. Psychological Science, 5, 253-259.

Peterson, M. A., \& Gibson, B. S. (1994b). Object recognition contributions to figure-ground organization: Operations on outlines and subjective contours. Perception \& Psychophysics, 56, 551-564.

Peterson, M. A., Harvey, E. H., \& Weidenbacher, H. L. (1991). Shape recognition inputs to figure-ground organization: Which route counts? Journal of Experimental Psychology: Human Perception and Performance, 17, 1075-1089.

Peterson, M. A., \& Kimchi, R. (1994). Object recognition contributions to figure-ground organization: Operations on outlines and subjective contours. Perception and Psychophysics, 56(5), 551-564.

Peterson, M. A., \& Lampignano, D. L. (2003). Implicit memory for novel figure-ground displays includes a history of border competition. Journal of Experimental Psychology: Human Perception and Performance, 29, 808-822.

Peterson, M. A., \& Salvagio, E. (2008). Inhibitory competition in figureground perception: Context and convexity. Journal of Vision, 8, 113.

Peterson, M. A., \& Skow, E. (2008). Inhibitory competitionbetween shape properties in figure-ground perception. Journal of Experimental Psychology: Human Perception and Performance, 34, 251-267.

Peterson, M. A., Mojica, A. J., Salvagio, E. \& Kimchi, R. (2017). Figural properties are prioritized for search under conditions of uncertainty: Setting boundary conditions on claims that figures automatically attract attention. Attention, Perception, \& Psychophysics, 79, 180199.

Pizlo, Z. (2010). 3D shape: Its unique place in visual perception. Cambridge, MA: MIT Press.

Raymond, J. E., \& O'Brien, J. L. (2009). Selective visual attention and motivation: The consequences of value learning in an attentional blink task. Psychological Science, 20, 981-988.

Rehder, B., \& Hoffman, A. B. (2005). Thirty-something categorization results explained: Selective attention, eyetracking, and models of category learning Journal of Experimental Psychology: Learning, Memory, and Cognition, 31(5), 811-829. https://doi.org/10.1037/ 0278-7393.31.5.811

Rock, I., \& Fleck, F. (1950). A re-examination of the effects of monetary reward and punishment on figure-ground perception. Journal of Experimental Psychology, 40, 766-776.

Rouder, J., \& Morey, R. (2012). Default Bayes factors for model selection in regression. Multivariate Behavioral Research, 47, 877-903. https://doi.org/10.1080/00273171.2012.734737 
Rubin, E. (1915). Synsoplevede Figurer: Studier i psykologisk Analyse. Første Del [Visually experienced figures: Studies in psychological analysis. Part one]. Copenhagen, Denmark: Gyldendalske Boghandel, Nordisk Forlag.

Salvucci, D. D., \& Goldberg, J. H. (2000). Identifying fixations and saccades in eye-tracking protocols. Proceedings of the Eye Tracking Research and Applications Symposium (pp. 71-78). https://doi.org/10.1145/355017.355028

Sanguinetti, J. L., Allen, J. J., \& Peterson, M. (2014). The ground side of an object. Psychological Science, 25, 256-264.

Schafer, R., \& Murphy, G. (1943). The role of autism in a visual figureground relationship. Journal of Experimental Psychology, 32, 335343.

Smith, D. E. P., \& Hochberg, J. E. (1954). The effect of "punishment" (electric shock) on figure-ground perception. The Journal of Psychology: Interdisciplinary and Applied, 38, 83-87. https://doi. org/10.1080/00223980.1954.9712919

Vecera, S. P., \& Farah, M. J. (1997). Is visual image segmentation a bottom-up or an interactive process? Perception \& Psychophysics, 59, 1280-1296.
Vecera, S. P., Flevaris, A. V., \& Filapek, J. C. (2004) Exogenous spatial attention influences figure-ground assignment. Psychological Science, 15(1), 20-26.

Watson, P., Pearson, D., Most, S. B., Theeuwes, J., Wiers, R. W., \& Le Pelley, M. E. (2019). Attentional capture by Pavlovian rewardsignalling distractors in visual search persists when rewards are removed. PLOS ONE, 14, e0226284.

Wertheimer, M. (1938). Laws of organization in perceptual forms. In W. D. Ellis (Ed.), A source book of Gestalt psychology (pp. 71-94). London, England: Routledge \& Kegan Paul. (Original work published 1923)

Wilbertz, G., van Slooten, J., \& Sterzer, P. (2014). Reinforcement of perceptual inference: Reward and punishment alter conscious visual perception during binocular rivalry. Frontiers in Psychology, 5, 1377.

Publisher's note Springer Nature remains neutral with regard to jurisdictional claims in published maps and institutional affiliations. 